\title{
On Modelling of Data-Driven Monotone Zero-Order TSK Fuzzy Inference Systems using a System Identification Framework
}

\author{
Chin Ying Teh, Yi Wen Kerk, Kai Meng Tay, and Chee Peng Lim
}

\begin{abstract}
A system identification-based framework is used to develop monotone fuzzy If-Then rules for formulating monotone zero-order Takagi-Sugeno-Kang (TSK) Fuzzy Inference Systems (FISs) in this paper. Convex and normal trapezoidal and triangular fuzzy sets, together with a strong fuzzy partition strategy (either fixed or adaptive), is adopted. By coupling the strong fuzzy partition with a set of complete and monotone fuzzy If-Then rules, a monotone TSK FIS model can be guaranteed. We show that when a clean multi-attribute monotone data set is used, a system identification-based framework does not guarantee the production of monotone fuzzy If-Then rules, which leads to non-monotone TSK FIS models. This is a new learning phenomenon that needs to be scrutinized when we design data-based monotone TSK FIS models. Two solutions are proposed: (1) a new monotone fuzzy rule relabeling-based method; (2) a constrained derivative-based optimization method. A new modelling framework with an adaptive fuzzy partition is evaluated. The results indicate that TSK FIS models with better accuracy (a lower sum square error) and a good degree of monotonicity (measured with a monotonicity test) are achieved. In short, the main contributions of this study are validation of the new learning phenomenon and introduction of useful methods for developing data-based monotone TSK FIS models.
\end{abstract}

Index Terms - Degree of monotonicity, monotone data, monotone fuzzy rules, monotone fuzzy rules relabeling, monotonicity test, strong fuzzy partition, system identification, TSK fuzzy inference system.

\section{INTRODUCTION}

$\mathrm{T}$ The Fuzzy Inference System (FIS) with fuzzy If-Then rules has been successfully applied to solve real life problems [1], [2]. Traditionally, fuzzy If-Then rules are normally constructed through knowledge acquisition from human experts, i.e., knowledge-driven FIS models. However, it is difficult and expensive to obtain fuzzy rules from experts. Furthermore, translating the experience of a human expert directly into fuzzy linguistic values is subject to human

Chin Ying TEH and Kai Meng TAY are with the Faculty of Engineering, University Malaysia Sarawak (e-mail: christineteh_10@yahoo.com and kmtay@unimas.my, tkaimeng@yahoo.com). Yi Wen KERK and Chee Peng LIM are with the Institute for Intelligent Systems Research and Innovation, Deakin University (e-mail: kerkyiwen@hotmail.com and chee.lim@deakin.edu.au). intuition. As a result, many methods to automatically generate fuzzy If-Then rules from numerical data, i.e., data-driven FIS models, have been proposed. Among the popular data-driven methods include system identification [2], gradient descent learning [3]-[6], fuzzy-neural [2], [7], fuzzy c-mean clustering [8], least-square [9], [10], and ad hoc methods (according to the terminology used in [11]) [4], [12]-[14].

\section{A. Monotonicity Property in FIS Modeling}

A model that obeys the monotonicity property between the input and output variables, i.e., a monotone model, is useful in various applications, e.g., a feedback system [15], a monotone control system [16], a biological system [17], and a risk priority model in Failure Mode and Effect Analysis (FMEA) [18]. As such, how to preserve the monotonicity property of FIS models constitutes an important research question. In this aspect, mathematical conditions that allow various FIS models to satisfy the monotonicity property are available in the literature, e.g., Mamdani FIS [19], TSK FIS [20]-[22], SIRM FIS [23] and interval type-2 FIS [24]. Besides that, studies on how to adopt these mathematical conditions to facilitate the FIS modelling procedure are reported [22], [25], [26]. Other investigations on the monotonicity property of fuzzy relational inference [27] and implicative-based fuzzy models [28] are also reported.

It is worth-noting that mathematic analyses [20], [21], [23], [24] have indicated that monotone fuzzy If-then rules offer an important building block to construct a monotone TSK FIS model. A monotone TSK FIS model is guaranteed to be produced when a strong fuzzy partition strategy (Definition 3) is used with a set of complete and monotone fuzzy If-Then rules (Definition 5) [20], [22], [29]. In [21], we showed that monotone fuzzy If-Then rules from human experts could be noisy, resulting in non-monotone fuzzy If-Then rules. Therefore, monotone fuzzy rule relabeling (MFRR) methods to pre-process non-monotone fuzzy If-Then rules from human experts are devised for constructing TSK FIS models.

\section{B. Motivations and Aims}

In [30]-[32], we examined the construction of a monotone TSK FIS model from data samples. Indeed, generating monotone fuzzy If-Then rules using data-driven methods is a new research direction. In this paper, a monotonicity test to evaluate the monotonicity property of an FIS model with a degree of monotonicity (DOM) is established (Section II (B)). Besides that, the definition of monotone data (Definition 6) is provided.

Specifically, we focus on data-driven monotone zero-order TSK FIS modelling using a system identification-based 